Kosslyn's idea is clearly derivative of Dawkins' theory of "the extended phenotype".

The two chapters of Section VI are integrative. Kimberley and Wolpe discuss the ethical implications of evolutionary cognitive neuroscience (Chapter 20). The reader of this chapter comes away with a good understanding of the fears generated by EP approaches to cognition, for example, concerns that murderers will not be held responsible for heinous acts. The Editors wrap things up in Chapter 21. They emphasize the importance of cross-fertilization between genetics, cognitive psychology, archaeology, anthropology, and primatology as the fuel driving major theorizing and advances.

Clinical neuropsychology could use the cross-fertilization that EP provides. The oft-repeated truism that "psychology gives itself away" applies yet again. How many readers have heard of psychologist Harry J. Jerison and his Enceph- alization Quotient (EQ)? This ratio of observed brain to expected brain volume (based on body size) is an analytic tool widely employed in many fields, but not in psychology. Evolutionary Cognitive Neuroscience is best suited for academic neuropsychologists or those clinicians at a lifestage where they desire to reflect on theory. I guarantee parts of this book will be unnerving to those who resist certain scientific ideas because of deeply held egalitarian or religious impulses. But science is antidemocratic; good ideas are not proven by a show of hands.

\section{REFERENCES}

Hamilton, Richard (1964). The evolution of social behavior. Journal of Theoretical Biology, 7, 1-16.

Zimmer, C (2007, April 3). Time in the animal mind. New York Times.

\title{
All the Important Things You Wanted to Know About the Effects of Stroke: Location, Location, Location
}

DOI: $10.1017 /$ S1355617708080235

\author{
The Behavioral and Cognitive Neurology of Stroke. 2007. Olivier Godefroy and Julien \\ Bogousslavsky (Eds.). Cambridge, UK: Cambridge University Press, 664 pp., \$160.00 (HB) \\ Reviewed by Steven Mattis, Ph.D., ABPP/CN, Clinical Professor of Neuropsychology in Neurology, \\ Weill-Cornell College of Medicine and Private Practice, White Plains, New York, USA
}

The Behavioral and Cognitive Neurology of Stroke is a very handy reference book for the bedside or brief office examination of the stroke patient. The initial chapter highlights the need for serial mental status exams during the critical initial post stroke period. The book is then organized by functional sections, each containing several chapters. The sections are organized by neuropsychological processes in the usual "walk around the brain" format. Thus, the reader is offered sections on motor and gestural disorders, aphasia and arthric disorders, hemineglect and right hemisphere syndromes, agnosia and Balint's syndrome, and executive and memory disorders. Of great significance is the inclusion of a section on behavioral and mood disorders, which reviews the clinical domains where the disciplines of psychiatry and neurology overlap. The final section, dementia and anatomical left/ right syndromes, extends and integrates the previous sections.

Given the title, the reviewer had expected the sections to be organized by vascular systems, e.g., behavioral and cognitive manifestations of stroke of the anterior communicating artery. However, after the walk about the brain, there is a final chapter, Right Versus Left Hemisphere Syndromes, by Isabel P. Martins and colleagues describing the common cluster of neuropsychological findings observed with vesselspecific obstructive strokes. Most chapters are clearly written, succinct, and have the added advantage of containing several tabular sections termed "Key points" which provide a summary of the section's most salient information. Most chapters describe easy to administer, valid procedures to assess a specific neuropsychological process and contain a brief section, perhaps several paragraphs, highlighting rehabilitative or treatment programs for enhancement of the disorders discussed.

The chapters are generally well researched and well written. Each presents a succinct review of the literature, and offers what is the present common wisdom concerning the phenomenology, the underlying clinical-pathological correlations, and the treatment for each disorder. The chapter on dysarthria by Pascal Auzou is an excellent example of the ability to present a brief, 16 page, but authoritative review of a domain, including an intelligent review of the literature supporting various treatment approaches. Not all the clinical entities lend themselves well to such a tight format. The chapter on depression after stroke, by Carota and Paolucci, is perhaps the best exemplar of such difficulties. The authors struggle to reconcile psychiatry's descriptive diagnostic criteria of disorders of behavior, affect, and thought with neurology's clinical-pathological criteria for such disorders. The two diagnostic models do not allow for a simple agreement as to what constitutes a diagnosis. The authors question the psychiatric distinction between the diagnoses of depression and depression occurring with a chronic medical condition, and the distinction between endogenous versus reactive depression. These distinctions become critical as one attempts to reconcile the conflicting findings in the 
literature relating neuroanatomic systems and depression. The chapter contains little gems like the inclusion of scales appropriate for the assessment of depression in aphasic patients and is an excellent chapter for a brief review of a complex subject.

The chapter by Dieguez, Staub, and Bogousslavsky concerning asomatognosia was both the most interesting and least scientifically satisfying. It is among the longest chapters, 36 pages, including an appendix entitled "A funneled guideline for the bedside interview". The authors present the reader with a diagnostic entity termed an asomatognosia which they state is "roughly defined as the disturbances of the body schema, (and) encompasses a wide array of clinical pictures under a unitary conceptual framework" (p. 215). The authors note that usually asomatognosia is viewed as a subsection of the study of anosognosia or neglect. However, they propose to return to the French tradition, that of Lhermitte (1939) and Hecaen \& de Ajuriaguerra (1952) in which anosognosia was viewed as an instance of hemiasomatognosia. The authors then present a very detailed and well reasoned explication of their framework for disorders of body schema. However, the level of detail contained in the framework far outstripped the known loci of lesions causing each of the detailed phenomena. The reader is left, therefore, with a "think piece" concerning the nature and etiology of disorders of body schema with very little clinicalpathological correlative data to support the reliability and validity of the constructs. Overall, while this chapter offers the reader an impressively logical framework, the level of authoritative literature supporting the validity of the framework was well below the level offered in the other chapters.

In summary, The Behavioral and Cognitive Neurology of Stroke is an excellent source book. It is likely to be re-read many times by its owner; indeed, every time the clinician is requested to see an "interesting" patient and needs a fast review of the phenomenology, assessment procedures, neuroanatomic implication, and appropriate treatment plan for the patient he/she is about to encounter.

\section{REFERENCES}

Lhermitte, J. (1939). L'Image de Notre Corps. Paris: Nouvelle Revue Critique.

Hecaen, H. \& de Ajuriaguerra, J. (1952). Meconnaissances et Hallucinations Corporelles. Integration et disintegration de la somatognosie. Paris: Masson.

\section{What's New With the Frontal Lobes?}

DOI: $10.1017 /$ S1355617708080247

\section{The Human Frontal Lobes: Functions and Disorders (Second Edition). 2007. Bruce L. Miller \& Jeffrey L. Cummings (Eds.). New York: The Guilford Press, 666 pp., \$95.00 (HB) \\ Reviewed by Vicki Anderson, Ph.D., Department of Psychology, Royal Children's Hospital, Parkville, Victoria, Australia}

Miller and Cummings published their original text, The Human Frontal Lobes: Functions and Disorders, in 1999. At that time, their work was quite unique and provided a valuable reference for both health professionals and researchers in the emerging field of the frontal lobes. The years since then have seen an increasing focus on these fascinating brain structures across a range of disciplines including neurosciences, neurology, neuropsychology and psychiatry. The importance of the frontal structures for both cognition and emotion is firmly established and the complexity of these associations continues to challenge those working in these fields. As Miller and Cummings note, due to the major advances that have occurred since they first published their work, their second edition represents not only an update but also an introduction to a range of new issues. In particular, this second edition emphasizes advances in neurosciences and neuroimaging with a substantial amount of new material presented in these areas. In addition, the increasing interest in the role of the frontal lobes in social and emotional function is also acknowledged and discussed.

The text is divided into an introduction and six content areas: anatomy, neurochemistry, neuroimaging, neuropsy- chology, neurology and neuropsychiatry. Chapters vary from highly research focused to clinical in nature, but with an underlying emphasis on providing an evidence-base for the reader. The text benefits from the joint neurological and psychiatric expertise of the authors, and there is an attempt throughout to link these two areas, as well as to relate basic science with clinical issues.

The introductory sections of the text illustrate the breadth of knowledge of the editors. They provide a chronological account of the key factors which have facilitated the study of the frontal lobes, starting with the well known tale of Phineas Gage, and moving through a number of influential advances in the field, noting conceptual leaps, such as the emergence of the theory of working memory, as well as the gradual acknowledgment of the importance of the frontal lobes to a wide range of clinical conditions, such as dementias, depression and schizophrenia. The changing nature of the field is highlighted, with the frontal lobes and their functions initially viewed as largely uni-dimensional, but with a more contemporary perspective gradually emerging in keeping with scientific advances, and taking on a more multi-dimensional systemic framework. These chapters also 